\title{
IMPACTO DE LA ORIENTACIÓN PROACTIVA Y REACTIVA AL MERCADO SOBRE EL ÉXITO DE LA INNOVACIÓN. EL PAPEL MODERADOR DE LA ORIENTACIÓN AL APRENDIZAJE*
}

\author{
Fabián F. Osorio Tinoco ** \\ Miguel Hernández Espallardo*** \\ Héctor Augusto Rodríguez Orejuela ${ }^{* * * *}$
}

doi:10.11144/Javeriana.cao27-49.iopr. Este artículo hace parte del proyecto de investigación “Antecedentes y resultados de la innovación en la industria manufacturera colombiana (C.I. 8164) y ECO2013-47969-P (Ministerio de Economía y Competitividad, España) y de la tesis doctoral “Antecedentes y resultados de la orientación al mercado reactiva y proactiva y el papel moderador del entorno y el aprendizaje". El artículo se recibió el 11/06/2014 y se aprobó el 01/12/2014. Sugerencia de citación: Osorio Tinoco, F., Hernández-Espallardo., M. y Rodríguez Orejuela, H. A. (2014). Impacto de la orientación proactiva y reactiva al mercado sobre el éxito de la innovación. El papel moderador de la orientación al aprendizaje. Cuadernos de Administración, 27 (49), 109-133. http://dx.doi.org/10.11144/ Javeriana.cao27-49.iopr

** Candidato a Doctor en Administración de la Universidad del Valle, Cali, Colombia. Profesor Asistente de la Universidad Javeriana, Cali, Colombia. Correo electrónico: fosorio@javerianacali.edu.co

*** Doctor en Ciencias Económicas y Empresariales de la Universidad de Murcia, Murcia, España, 1996. Docente del Departamento de Comercialización e Investigación de Mercados de la Universidad de Murcia, Murcia, España. Correo electrónico: migher@um.es

***** Doctor en Ciencias de Empresa de la Universidad de Murcia, Murcia, España, 2004. Docente de la Universidad del Valle, Cali, Colombia. Correo electrónico: augusto.3030@hotmail.com 


\section{Impacto de la orientación proactiva y reactiva al mercado sobre el éxito de la innovación. El papel moderador de la orientación al aprendizaje}

\section{RESUMEN}

Desde la perspectiva teórica propuesta por Narver et al. (2004), este artículo analiza el efecto de la orientación al mercado reactiva y proactiva sobre el éxito de la innovación y la moderación de la orientación al aprendizaje con fuentes internas y de mercado. Utilizando regresión lineal, se encuentra que la orientación al mercado reactiva tiene un efecto lineal positivo sobre el éxito de la innovación, mientras la proactiva tiene un efecto de $\mathrm{U}$ invertida, moderado por la orientación al aprendizaje de mercado. El texto demuestra que el efecto de cada tipo de orientación depende del tipo aprendizaje. Y se concluye que las empresas mejorarán su éxito de la innovación combinando adecuadamente su orientación al mercado con el tipo de aprendizaje utilizado.

Palabras clave: Innovación, orientación al mercado, aprendizaje organizacional.

Clasificación JEL: D40, M10, M30

\section{The impact of proactive and reactive market orientation on innovation success. The moderating role of learning orientation}

\section{Abstract}

From the theoretical perspective of market orientation proposed by Narver et al. (2004), this paper analyzes its relationship with innovation success and the moderating role of market and internally focused learning orientation. By ordinary least squares estimation of linear regression function it was found that reactive market orientation has a positive linear effect on innovation success. Meanwhile, proactive market orientation has an inverted $U$ shaped effect that is moderated by market focused learning orientation. The paper demonstrates that each orientation type effects depend on the learning process type. It therefore follows that the enterprise's innovation success would have better results when combining an adequate market orientation and the learning process type applied.

Keywords: Innovation, market orientation, learning orientation.

\section{Impacto da orientação proativa e reativa no mercado sobre o sucesso da inovação. O papel moderador da orientação na aprendizagem}

\section{RESUMO}

Sob a perspectiva teórica proposta por Narver et al. (2004), analisa-se o efeito da orientação proativa e reativa no mercado sobre o sucesso da inovação e a moderação da orientação na aprendizagem com fontes internas e de mercado. Utilizando regressão linear, constata-se que a orientação reativa no mercado tem um efeito linear positivo sobre o sucesso da inovação, enquanto a proativa tem um efeito de $U$ invertido, moderado pela orientação na aprendizagem de mercado. Este texto demonstra que o efeito de cada tipo de orientação depende do tipo de aprendizagem. Conclui-se que as empresas melhorarão seus resultados de inovação ao combinarem adequadamente sua orientação no mercado com o tipo de aprendizagem utilizada.

Palavras-chave: Inovação, orientação no mercado, aprendizagem organizacional.

Classificação JEL: D40, M10, M30 


\section{Introducción}

La innovación constituye una importante fuente de ventaja competitiva (Song y Thieme, 2006). Sin embargo, para que ello se dé es necesario inculcar una cultura de búsqueda constante de oportunidades que impacte la calidad de dicha innovación. El éxito de la innovación, entendido como una salida positiva del proceso de innovación, es el principal medio por el cual las firmas mantienen y expanden sus mercados de productos y clientes (Baker y Sinkula, 2009; Simpson et al., 2006). Esto implica la necesidad de prestar una atención especial a este asunto por parte de la alta dirección para el logro de los objetivos de desempeño superior en las empresas. Con el fin de aumentar los niveles de éxito de la innovación, la literatura sugiere que la orientación al mercado es muy importante, por el efecto que puede generar sobre la capacidad de innovar y el desempeño organizacional (AtuaheneGima, 1995; Deshpande et al., 1993; Han et al., 1998; Jaworski y Kohli, 1993; Li y Calantone, 1998; Pelham y Wilson, 1996; Slater y Narver, 1994). Una orientación organizacional hacia el mercado se basa en una cultura orientada externamente que actúa sobre la creencia de que toda decisión comienza con el mercado y la anticipación de oportunidades para lograr una ventaja (Webster, 1992).

A pesar de la gran cantidad de estudios que sugieren que la orientación al mercado está asociada positivamente a un desempeño superior en formas tales como el número de innovaciones adoptadas (Han et al., 1998), el rendimiento (Hult et al., 2004), el desem- peño de programas de desarrollo de nuevos productos (Atuahene-Gima et al., 2005) y el éxito de nuevos productos (Narver et al., 2004), aún no hay consenso sobre el papel de la orientación al mercado en el éxito de la innovación. Por ejemplo, algunos investigadores proponen que una empresa que se orienta al mercado puede disminuir su capacidad para innovar si sólo escucha la voz de los consumidores (Christensen y Bower, 1996; Lukas y Ferrell, 2000), o por la presencia de miopía en las actividades de investigación y desarrollo (Frosch, 1996).

Narver et al. (2004) proponen que el desacuerdo acerca de la relación entre la orientación al mercado y el éxito de la innovación se da por una estrecha comprensión de la orientación al mercado, la cual se ha concebido tradicionalmente como una orientación solo reactiva, que consistiría en atender las necesidades de los clientes. Además, dicho estudio establece que una completa orientación al mercado también consta de un modo proactivo que concierne al descubrimiento y satisfacción de las necesidades futuras no manifestadas espontáneamente por los clientes. A pesar de la propuesta de una orientación al mercado más completa para entender la relación con la innovación como la presentada por Narver et al. (2004) hasta hoy los investigadores no han explicado ni puesto atención de manera suficiente a la naturaleza de las dimensiones proactiva y reactiva sobre el éxito de la innovación (Grinstein, 2008).

Para abordar dicho desacuerdo, la perspectiva del aprendizaje organizacional ha propuesto que la orientación al aprendizaje es 
necesaria para prevenir que una orientación al mercado sea excesiva en centrarse en el cliente (Baker y Sinkula, 2007). La orientación al aprendizaje se define como la capacidad de la empresa para adquirir, diseminar, desaprender y usar la información externa y/o interna para el cambio organizacional (Weerawardena et al., 2006). Si bien la literatura de aprendizaje y de orientación al mercado ha estudiado de manera importante la integración de estos dos conceptos con relación a la innovación (Baker y Sinkula, 1999a; Grinstein, 2006; Yannopoulos et al., 2012), son escasos los estudios que integren las dos vertientes de la orientación al mercado (reactiva y proactiva) y diferentes formas de aprendizaje con el éxito de la innovación. A pesar de que Baker y Sinkula (1999b) propusieron un efecto de moderación de la orientación al aprendizaje sobre la relación entre la orientación al mercado y el éxito de un nuevo producto, hay poca evidencia sobre dicho efecto con diferentes tipos de aprendizaje y las vertientes reactiva y proactiva de la orientación al mercado.

El propósito de este estudio es llenar estos vacíos y arrojar nuevas luces sobre cómo el éxito de la innovación puede mejorarse cuando actúan diferentes modos de orientación al mercado (reactiva y proactiva) moderados por la orientación al aprendizaje basado en fuentes de mercado y fuentes internas. Mientras que estudios previos establecen que ambas vertientes de la orientación al mercado son importantes para el éxito de la innovación (Narver et al., 2004; Slater y Narver, 1998; Tan y Liu; 2014; Zhang y Duan, 2010), pretendemos indagar el efecto diferencial de la adopción de una orientación al mercado proactiva y reactiva sobre el éxito de la innovación. Este artículo también llama la atención sobre la importancia que tiene el aprendizaje organizacional en el modo como la orientación proactiva influye en el éxito de la innovación.

A continuación presentamos los conceptos principales y las hipótesis que los relacionan. Continuamos con la descripción de la metodología utilizada para la recolección de datos que permite contrastar empíricamente dichas hipótesis. Seguidamente exponemos la metodología de análisis de datos y los resultados obtenidos. Concluimos este trabajo con la discusión de los resultados y sus implicaciones.

\section{Marco teórico y desarrollo de hipótesis}

La innovación tiene que ver con el desarrollo, la producción y la comercialización de una invención, así como la difusión y adopción de dicha novedad por parte de los clientes (García y Calantone, 2002). También puede consistir en la implantación de novedades de proceso y administrativas (Danneels y Kleinschmidt, 2001). La innovación es importante porque permite a las empresas poder aprovechar oportunidades de crecimiento y expansión a nuevas áreas de negocio. Por una parte, es un proceso que compromete el desarrollo tecnológico combinado con la introducción al mercado de la invención. Por otra, dicho proceso es iterativo, es decir, incluye una primera introducción de una nueva innovación y la reintroducción de una innovación mejorada. Este proceso iterativo implica diferentes tipos de innovación lla- 
mados tradicionalmente innovación radical e innovación incremental.

Las empresas requieren de una estructura y conocimiento focalizado en el desarrollo de competencias organizacionales clave que les permitan el despliegue de recursos, tecnologías, operaciones y mercados, a fin de que los resultados de los procesos de innovación sean exitosos. Por tanto, los procesos de innovación deben estar en un sistema organizacional que provea soporte para la generación continua de nuevas ideas y productos (Hurley et al., 2005). Así, los resultados exitosos de dicho proceso no deberían limitarse exclusivamente a observar el número de innovaciones que la organización adopte, como generalmente se ha estudiado (García y Calantone, 2002; Simpson et al., 2006). Mientras que numerosos estudios han examinado el éxito de la innovación con variables como la tasa, el número y el tipo de innovación (Han et al., 1998; Hult et al., 2004; Narver et al., 2004) pocos estudios han contemplado el éxito de la innovación como una consecuencia estratégica más amplia, más allá del simple conteo de las innovaciones (Ruvio et al., 2014; Simpson et al., 2006; Totterdell et $a l ., 2002)$. Por esta razón nos basamos en el trabajo de Simpson et al. (2006) en el cual el éxito de la innovación es el resultado del desarrollo e implementación de una orientación de la firma hacia la innovación, cuyas consecuencias se constituyen no solamente de las innovaciones que una firma produce sino también de las ventajas y beneficios que consigue del mercado (relacionadas con los clientes y la competencia). Así, este estudio contribuirá a la literatura al tener en cuenta una perspectiva más amplia para determinar el éxito de los esfuerzos de las empresas que desarrollan procesos de innovación.

Una de las preguntas que persiste en la investigación sobre innovación es cómo la orientación al mercado impacta los resultados de innovación. Si bien la influencia de orientarse al mercado está ligada a las capacidades de procesamiento de información para identificar y responder rápidamente a las necesidades actuales y futuras dando lugar a la introducción de nuevos productos (Day, 1994b; Kohli y Jaworski, 1990), existen diferentes posturas sobre la relación entre la orientación al mercado y la innovación. Por una parte, algunos autores proponen que se requiere de manera constante una fuerte orientación al mercado para participar de manera activa en la innovación (Day, 1994b; Kohli y Jaworski, 1990; Slater y Narver, 1999). Otros proponen que la orientación al mercado llevará a las empresas a sobreponderar lo que el cliente proponga en detrimento de la "verdadera" innovación (Christensen y Bower, 1996; Lukas y Ferrell, 2000). Una tercera postura propuesta desde la perspectiva del aprendizaje organizacional establece que otros factores culturales, como la orientación al aprendizaje, son necesarios para impedir que la orientación al mercado esté centrada en el cliente (Baker y Sinkula, 1999a; Baker y Sinkula, 1999b; Slater y Narver, 1999).

Para aclarar la falta de consenso sobre el papel de la orientación al mercado y el éxito de la innovación, Narver et al. (2004) proponen una perspectiva de la orientación al mercado de doble constructo. Describen 
dos tipos de orientación al mercado: reactiva y proactiva. Una orientación al mercado reactiva se centra en el conocimiento y experiencia actual que la empresa tiene del cliente, lo que le permite una profunda comprensión y satisfacción de las necesidades actuales (expresas) (Atuahene-Gima et al., 2005). Las necesidades expresas hacen referencia a aquello de lo que el mercado es consciente (Narver et al., 2004). De otro lado, la orientación al mercado proactiva se centra en descubrir, en entender y satisfacer necesidades futuras (latentes) de los clientes, observando el comportamiento en un contexto de identificar nuevas oportunidades de mercado (Atuahene-Gima et al., 2005). Las necesidades latentes hacen referencia a aquello de lo que el mercado aun no es consciente (Narver et al., 2004). Son escasos los estudios previos sobre la relación de la orientación al mercado reactiva y proactiva con el éxito de la innovación, y la necesidad de profundizar en su naturaleza ha sido recientemente enfatizada por más investigadores (Grinstein, 2008; Lamore et al., 2013; Yannopoulos et al., 2012). Los estudios desarrollados hasta el momento difieren sobre la naturaleza de la orientación al mercado reactiva y proactiva con la innovación. Por ejemplo, la mayoría de los estudios reconocen una naturaleza lineal de ambas orientaciones con los resultados de la innovación (Lamore et al., 2013; Li et al., 2008; Narver et al., 2004; Voola y O'Cass, 2010; Yannopoulos et al., 2012; Zhang y Duan, 2010). Sin embargo, hay algunos estudios cuyos resultados determinan funciones curvilíneas de ambas orientaciones al mercado (Atuahene-Gima et al., 2005; Tsai et al.,
2008). Pese a estos avances, los resultados, además de carecer de evidencia estadística en algunos casos (Atuahene-Gima et al., 2005), en otros difieren en determinar si la función curvilínea es positiva y/o negativa (Tsai et al., 2008).

Por otra parte, aunque Baker y Sinkula (1999b) intentaron probar el efecto sinérgico entre la orientación al mercado y el aprendizaje sobre el éxito de la innovación, existe poca evidencia con respecto a la interacción de estos dos conceptos utilizando diferentes tipos de aprendizaje y de vertientes de la orientación al mercado (Yannopoulos et al., 2012). El propósito de este estudio es contribuir a la explicación de la naturaleza de la relación entre la orientación al mercado y el éxito de la innovación, y arrojar nuevas luces sobre cómo mejorar los resultados de la innovación. Nuestro estudio propone que las orientaciones al mercado reactiva y proactiva interactúan de formas diferentes. A continuación analizamos los efectos de la orientación al mercado reactiva y proactiva sobre el éxito de la innovación.

\subsection{Orientación al mercado reactiva y el éxito de la innovación}

La orientación al mercado comprende un valor basado en la filosofía estratégica, que se manifiesta en comportamientos diseñados para mantener a la empresa cerca al consumidor (Moorman y Rust, 1999). La orientación al mercado refleja el grado en el cual las empresas establecen las necesidades y deseos de los clientes como un principio organizacional (Jaworski y Kohli, 1993). 
Los hallazgos convencionales apuntan a que la orientación del mercado reactiva, en general, tiene un efecto positivo sobre el desempeño del desarrollo de productos y/o el éxito de la innovación (Atuahene-Gima et al., 2005; Baker y Sinkula, 2009; Narver et al., 2004). Las empresas con una fuerte orientación al mercado reactiva escuchan las necesidades actuales de los consumidores y reaccionan mediante el desarrollo de procesos que permitan el ajuste de las líneas de productos que satisfagan esas necesidades (Baker y Sinkula, 2009). Una empresa que realiza innovaciones de acuerdo con este enfoque hace seguimiento a los requerimientos del cliente para desarrollar nuevos productos y/o servicios o para adaptar los procesos internos según los requerimientos explícitos realizados por los clientes.

Por otra parte, los estudios previos sobre la orientación al mercado reactiva han mostrado evidencia de que tiene un efecto saturación sobre el desempeño (Atuahene-Gima et al., 2005; Tsai et al., 2008). Es decir, a pesar de las ventajas que ofrece la familiaridad con el dominio operacional asociado a la orientación al mercado reactiva ocurre también que este efecto positivo pierde vigor a partir de un nivel que ya se puede considerar como de saturación (Cohen y Levinthal, 1990; Levinthal y March, 1993). Una empresa considerará en una primera instancia las necesidades más evidentes e importantes de los clientes. Sin embargo, de acuerdo con Narver et al. (2004), las empresas que se centran en satisfacer este tipo de necesidades hasta el extremo, por un lado, irán progresivamente dedicando más recursos a la satisfacción de "caprichos" de los compradores con menor efecto sobre el desempeño; por otro, pueden limitar la atracción y generar esfuerzos insuficientes para retener a los clientes. En este sentido los beneficios ofrecidos pueden ser conocidos fácilmente por los competidores, lo que los llevaría de manera natural a ofrecer las mismas ventajas siendo necesario ingresar en una competencia de precios en el intento de crear valor superior para los clientes. Por lo tanto, un exceso de orientación al mercado reactiva ya no impactará positivamente el éxito de la innovación. Se propone entonces que:

H1: El efecto de la orientación al mercado reactiva sobre el éxito de la innovación sigue una forma de " $U$ " invertida $(\cap)$. Así, tiene efectos positivos para niveles bajos que decrecen significativamente conforme aumenta dicha orientación.

\subsection{Orientación al mercado proactiva y el éxito de la innovación}

De acuerdo con Narver et al. (2004) las necesidades latentes son universales, existen en cada cliente y pueden ser descubiertas por una empresa que se comprometa en focalizarse y buscar disciplinadamente, mediante la observación cuidadosa de los comportamientos de los clientes, las posibles soluciones a los problemas que enfrentan. Por lo tanto, para que una empresa mejore los resultados de la innovación debe ir más allá de las necesidades actuales o conscientes de los clientes intentando descubrir sus necesidades futuras (latentes). Esto requiere que continuamente la empresa exceda las expectativas de sus clientes. De otra parte, una orientación al mercado proactiva alertará a 
la empresa de nuevos mercados y refleja un comportamiento de aprendizaje exploratorio que involucra la búsqueda de nueva y diversa información de necesidades que los clientes aún no hacen consciente (Atuahene-Gima et al., 2005; Narver et al., 2004). Esto hace suponer un efecto positivo de la orientación al mercado proactiva sobre el éxito de la innovación.

Sin embargo hay varios matices que pueden aducirse como influyentes en la forma definitiva que dicho efecto puede tener. Al igual que ocurría en el caso de la orientación al mercado reactiva, hay motivos para pensar en un efecto saturación. Un exceso de orientación proactiva puede conllevar una inversión excesiva en mercados de gran potencial pero todavía no realizados, con un altísimo componente de riesgo debido al grado de ineficiencia asociado a conocimiento e información no familiar (Atuahene-Gima et al., 2005). Por otro lado deberán hacerse enormes esfuerzos que implica la adaptación de las estructuras de la organización (Boumgarden et al., 2012). Una orientación al mercado proactiva requiere que la empresa se focalice en la búsqueda de información de necesidades sin descubrir de los clientes, requiriendo habilidades que van más allá de las conseguidas por la firma a través de la experiencia y experimentación, exigiendo el involucramiento en proyectos que no permiten explotar los conocimientos y experiencias previas sobre el mercado y que requieren afrontar información y conocimiento que están más allá del alcance de los procesos y actividades de procesamiento de información y de desarrollo de productos. Esta sobrecarga de información y nuevo co- nocimiento incrementa la incertidumbre en la coordinación de los proyectos enfrentándose a ajustes organizacionales que pueden restringir, contradecir o interferir con otros generando costos y riesgo adicionales. Así, la adquisición de información que dista mucho de las necesidades actuales de los clientes y la incertidumbre propia de las necesidades futuras podrían limitar el éxito de las innovaciones. Por lo tanto se propone que:

H2: El efecto de la orientación al mercado proactiva sobre el éxito de la innovación sigue una forma de " $U$ " invertida ( $\cap$ ). Así, tiene efectos positivos para niveles bajos que decrecen significativamente conforme aumenta dicha orientación.

La $\mathrm{H} 2$ propone un efecto de la orientación proactiva que es positivo aunque con saturación. Sin embargo, la literatura ha encontrado dificultades para contrastar empíricamente la presencia de dicho efecto positivo. Las afirmaciones anteriores realizadas para su justificación marcan una importante diferencia entre la orientación al mercado reactiva y la proactiva. Esta última es más arriesgada e incierta, más alejada de información explícita y, por tanto, el efecto positivo propuesto en H2 estará matizado por la presencia de algunas condiciones moderadoras.

La orientación al mercado proactiva implica un enfoque de experimentación, de riesgo asociado, de búsqueda de oportunidades en las necesidades insatisfechas y el desarrollo de innovaciones radicales. De acuerdo con la literatura, estas características son compartidas por una estrategia de aprendizaje organizacional por lo que, para reducir la in- 
certidumbre y aprovechar las oportunidades con mayor éxito, las empresas deben aprender a explotar sus conocimientos o adquirir nuevos de fuentes externas (March, 1991). Así, el éxito en la implementación de una orientación al mercado proactiva parece más acorde a la interacción con una orientación de la empresa hacia el aprendizaje.

Hunt y Morgan (1996) proponen que el aprendizaje es un importante recurso de la empresa que puede crear ventaja competitiva. La orientación al aprendizaje tiene que ver con el grado de compromiso de las empresas en cuestionar de manera ordenada y sistemática las creencias y prácticas fundamentales que definen los procesos de innovación (Baker y Sinkula, 1999b; Day, 1994a). Este recurso de orden superior es requerido por la empresa para adquirir, diseminar, descartar y usar el conocimiento y la nueva información, de manera que satisfaga sus modelos mentales y lógicas dominantes. Una empresa con una orientación al aprendizaje requiere de empleados que constantemente cuestionen las normas organizacionales que guían sus actividades y acciones influyendo sobre las actividades de procesamiento de información (Day, 1991; Sinkula et al., 1997).

Mientras que la orientación al mercado es un aspecto organizacional que dirige y prioriza las actividades de procesamiento de información del mercado, la orientación al aprendizaje incide en la propensión de la empresa a valorar y cuestionar dicha información (Baker y Sinkula, 1999b). Se ha argumentado que la orientación al mercado no es una condición suficiente para facilitar un tipo de innovación que cree una ventaja competitiva sostenible (Baker y Sinkula, 2002) y además se ha sugerido la necesidad de incorporar todas las formas de aprendizaje posibles en los procesos de innovación, incluyendo las diferentes fuentes de información (Weerawardena et al., 2006). La capacidad de aprendizaje es considerada como crítica tanto para la innovación como para la competitividad general de una empresa (Baker y Sinkula, 1999b). La literatura propone que una aproximación conjunta de la empresa hacia una orientación al mercado y una orientación al aprendizaje son claves para el éxito de la innovación (Dickson, 1996; Slater y Narver, 1995). Junto con el enfoque basado en el cliente las empresas deben inculcar la capacidad de participar en el aprendizaje si se quiere ser primero en el mercado con innovaciones exitosas (Baker y Sinkula, 1999b).

La literatura distingue varios enfoques de orientación al aprendizaje siendo dos muy destacados: el de la orientación al aprendizaje de mercado y la orientación al aprendizaje interno (Weerawardena et al., 2006). La orientación al aprendizaje de mercado es la capacidad de la empresa para adquirir, diseminar, descartar y usar conocimiento de mercado para la realización de cambios en la organización. Este aprendizaje de carácter generativo fundamentado en el mercado permite reducir los riesgos asociados a innovaciones radicales. Este tipo de innovación requiere exploración, riesgo y una continua búsqueda por necesidades insatisfechas de los clientes (Baker y Sinkula, 2007). Estos atributos son compartidos con una orientación al mercado proactiva y se puede argu- 
mentar que el aprendizaje generativo basado en el mercado es consistente tanto con la innovación radical como con la dimensión proactiva de la orientación al mercado, dado que permite la adquisición e interiorización del conocimiento del entorno externo de la empresa de manera que la empresa pueda aprender nuevas competencias más allá de sus límites actuales para lograr innovaciones radicales para nuevas categorías de producto que satisfagan las necesidades latentes de los clientes (Zhao et al., 2011). Una empresa que posee una mayor capacidad de aprendizaje de mercado no se ve limitada por una rígida e inflexible adhesión al modelo mental existente de procesamiento de la inteligencia de mercado. El desarrollo de un aprendizaje de mercado puede conducir a encontrar nuevos modelos mentales que posibiliten de manera más eficiente la búsqueda de información para la comprensión de necesidades latentes de los clientes, allanándose así el camino para avanzar en innovaciones más radicales (Baker y Sinkula, 2002). Si los miembros de la empresa cuentan con un nivel alto de orientación al aprendizaje de mercado, no solo van a generar y difundir la inteligencia de mercado, sino que también van a cuestionar constantemente la calidad de la información, la forma de interpretarla y de almacenarla a fin de validar la lógica que hay detrás de los aspectos que guían todo el procesamiento de información del mercado. De esa manera la calidad de los comportamientos asociados a la orientación al mercado proactiva necesariamente mejorarán. Por lo tanto se propone que:

H3: El aprendizaje basado en el mercado modera positivamente la relación entre la orientación al mercado proactiva y el éxito de la innovación. De modo que aumenta el efecto positivo en niveles bajos de orientación al mercado proactiva y disminuye su efecto negativo en niveles altos.

En cuanto a la orientación al aprendizaje interno, ésta consiste en la capacidad de una empresa para desarrollar conocimiento a través de sus recursos internos donde la $\mathrm{I}+\mathrm{D}$ juega un papel decisivo que incluye aprendizaje experiencial y aprendizaje experimental (Dixon, 1992; Huber, 1991). Estas actividades se interpretan como una experiencia de generación acumulativa de avances técnicos (Hyvarinen, 1990) y como una fuente clave en el logro de una innovación exitosa (Kim et al., 1993). Adicionalmente, la capacidad de una firma para absorber el conocimiento externo depende de sus esfuerzos en I+D. Dicha capacidad de absorción le permitirá a la firma realizar cosas diferentes. Las empresas que invierten en $\mathrm{I}+\mathrm{D}$ no solamente logran de manera directa nuevos desarrollos sino que también amplían y mantienen su capacidad de asimilar y explotar la información externa disponible (Cohen y Levinthal, 1989). El buen resultado del procesamiento de la inteligencia de mercado también depende de que los esfuerzos en I+D estén respaldados por lógicas de actuación adecuadas a la realidad y cercanía del mercado y que tanto actuaciones como resultados de dicha actividad basada en la experiencia sean lo más exacta posibles y compartida con toda la organización, conduciendo a la empresa hacia una mejora del procesamiento de la inteligencia de mercado y por lo tanto a innovaciones óptimas. Así se esperaría que el aprendizaje de fuentes internas modere 
positivamente la relación entre la orientación al mercado proactiva y el éxito de la innovación, al llamar la atención de la empresa a no seguir exclusivamente el enfoque y las señales de los consumidores, sino también a aprovechar el conocimiento interno para lograr identificar necesidades latentes producto de la investigación y desarrollo de la empresa. Por lo anterior se propone que:

\section{H4: El aprendizaje de fuentes internas mode-} ra positivamente la relación entre la orientación al mercado proactiva y el éxito de la innovación. De modo que aumenta el efecto positivo en niveles bajos de orientación al mercado proactiva y disminuye su efecto negativo en niveles altos.

\section{Metodología}

\subsection{Recolección de datos}

Los datos para este estudio se obtuvieron de una muestra de fabricantes colombianos. Mediante la triangulación de fuentes secundarias de información de tres instituciones nacionales se construyó un directorio de 1030 empresas. Dichas fuentes provienen, en primer lugar, de la segunda encuesta nacional de desarrollo e innovación tecnológica realizada por el Departamento nacional de planeación (DNP). De otra parte, de información del Departamento nacional administrativo de ciencia y tecnología (Colciencias) y por último del Departamento administrativo nacional de estadística (DANE). Una condición preliminar para depurar y construir la base de datos es el hecho de que la empresa hubiese estado implicada en, al menos, un proyecto de innovación considerado como significativo durante los últimos tres años (Lee y O'Connor, 2003). Con esta aproximación se contactó a una persona de la empresa con mayor conocimiento sobre el proyecto de innovación más significativo en el que la empresa hubiese estado involucrada durante dicho periodo.

Un total de 300 empresas cualificaban y estaban de acuerdo en participar, indicando la persona y la descripción del proyecto de innovación. Encuestadores profesionales $\mathrm{y}$ entrenados por los miembros del equipo de investigación fueron los encargados de contactar a la empresa y recoger los datos mediante entrevista personal. Esto reduce sustancialmente los problemas de control que ocurren al interior de las organizaciones (Cavusgil y Elvey-Kirk, 1998). Además en comparación con las encuestas por correo (Lee y O'Connor, 2003) o las encuestas en línea, este procedimiento ofrece una clara ventaja en términos de su tasa de respuesta (Olsen, 2009). A los encuestados se les recordó que no había respuestas buenas o malas; asimismo, que sus respuestas permanecerían anónimas y sin vincularlas con sus productos y/o empresas, minimizando la probabilidad de CMV. Para la prueba de este sesgo se utilizó la prueba de un factor de Harman. Se hace una prueba mediante un análisis factorial confirmatorio con un modelo de un factor en el que se cargan todas las variables observables utilizadas en este estudio. Este modelo mostró un $\chi^{2}=5960,69$ con 560 grados de libertad (comparado con el $\chi^{2}=$ 1374,15 con 550 grados de libertad del modelo de medición, ver cuadro 1). La prueba de la diferencia del Chi cuadrado sugiere un peor ajuste para el modelo unidimensional 
que para el modelo de medición (Anderson y Gerbing, 1988). El resultado de esta prueba confirma que el sesgo de método común no es problemático en nuestro estudio. Los encuestados corresponden al sexo masculino en la mayoría de los casos: $70,4 \%$ y ocupan el cargo de gerente general de la empresa o propietario en el 10,8\% de los casos, de director de producción: $40,3 \%$, de director de marketing: $12,4 \%$ y director de innovación o I+D en el $15,1 \%$ de los casos.

El total de empresas que constituyen la base de datos asciende a 296, lo que representa un margen de error de $\pm 4,8 \%$ con un nivel de confianza del $95 \%$ que pertenecen a una amplia gama de industrias manufactureras, con especial referencia al sector químico $(22,5 \%)$, fabricantes de caucho y plástico $(16,6 \%)$ y fabricantes de bebida y alimentos $(16 \%)$. La ventaja de la selección de un amplio y variado número de sectores es que permite una generalización de los resultados más allá de la naturaleza idiosincrática de una industria específica. Las empresas tienen en promedio 29,4 años de edad, con una facturación media de 15 millones de dólares y 305 empleados. En casi la mitad de los casos, el tipo de innovación es desarrollada en los productos: $47,6 \%$ y el $40,5 \%$ en los procesos productivos. En menor grado hay proyectos de innovación en la comercialización: 4,3\% y a nivel organizativo: 7,6\%.

\subsection{Escalas de medida}

Para la medición de los conceptos utilizados en este trabajo se han utilizado escalas multi-item extraídas de la literatura. Los enunciados utilizados, así como el rango de las respuestas se exponen en el cuadro 1 .

El éxito de la innovación es medido como una salida positiva de una orientación de la firma hacia la innovación, y cuyos ocho ítemes se constituyen de las innovaciones (número, tipo, velocidad y calidad) que una firma produce, y de las ventajas del mercado (relacionadas con los clientes y la competencia). Esta medida se basa en el trabajo de Simpson et al. (2006).

Las orientaciones al mercado reactiva y proactiva son medidas de acuerdo con las escalas desarrolladas por Narver et al. (2004). La primera evalúa el grado con el cual una firma logra entender y satisfacer las necesidades expresas de los clientes. La orientación al mercado proactiva evalúa el grado en el cual una empresa logra entender y satisfacer las necesidades latentes de los clientes.

Para la medición de los tipos de orientación al aprendizaje de acuerdo con sus fuentes de información, se utilizaron las medidas desarrolladas por Weerawardena et al. (2006). En el caso de la orientación al aprendizaje de mercado, entendida como el grado en que la empresa se compromete al aprendizaje a través de la adquisición de conocimiento sobre las preferencias del consumidor y el comportamiento del competidor, se usan ocho ítemes. Por último, la orientación al aprendizaje interno se mide mediante una escala de ocho ítemes que captura el grado en que la empresa genera conocimiento a través de fuentes internas de carácter experimental y experiencial de aprendizaje. 


\section{Cuadro 1. Resumen medición de variables}

\begin{tabular}{|c|c|c|c|}
\hline Descripción de los ítem de las escalas & $\begin{array}{l}\text { Carga de } \\
\text { factores }\end{array}$ & T-valor & $\begin{array}{l}\text { Fiabilidad } \\
\left(\mathrm{CR}^{\mathrm{a}} \alpha^{\mathrm{d}}\right)\end{array}$ \\
\hline $\begin{array}{l}\text { Éxito de la innovación (Fuente: Simpson et al., 2006) }{ }^{c} \\
\text { Calidad del resultado final } \\
\text { Éxito una vez en marcha } \\
\text { Utilidad de la innovación } \\
\text { Valor creado por la innovación para sus usuarios } \\
\text { Mayores beneficios para los usuarios finales de la innovación } \\
\text { Mejora de la imagen de la empresa } \\
\text { Ventaja estratégica frente a los competidores } \\
\text { Mejora en la competitividad de la empresa }\end{array}$ & $\begin{array}{l}0,56 \\
0,54 \\
0,57 \\
0,64 \\
0,64 \\
0,63 \\
0,60 \\
0,62\end{array}$ & $\begin{array}{r}9,63 \\
9,17 \\
9,69 \\
11,16 \\
11,27 \\
10,97 \\
10,37 \\
10,86\end{array}$ & $\begin{array}{l}C R=0,82 \\
\alpha=0,817\end{array}$ \\
\hline $\begin{array}{l}\text { Orientación al mercado reactiva (Fuente: Narver et al., 2004) }{ }^{\mathrm{b}} \\
\text { Constantemente monitoreamos nuestros niveles de compromiso con la atención de la } \\
\text { necesidad del cliente } \\
\text { Nuestra estrategia para la ventaja competitiva se basa en nuestra comprensión de la } \\
\text { necesidad del cliente } \\
\text { Medimos la satisfacción de los clientes de forma sistemática y frecuente } \\
\text { Estamos más enfocados en nuestros clientes que nuestros principales competidores } \\
\text { Creo que esta compañía existe principalmente para servir a los clientes } \\
\text { Los datos sobre satisfacción al cliente son diseminados en todos los niveles de la or- } \\
\text { ganización }\end{array}$ & $\begin{array}{l}0,67 \\
0,69 \\
0,62 \\
0,56 \\
0,62 \\
0,56\end{array}$ & $\begin{array}{r}11,93 \\
12,42 \\
10,85 \\
9,62 \\
10,96 \\
9,57\end{array}$ & $\begin{array}{l}C R=0,79 \\
\alpha=0,786\end{array}$ \\
\hline $\begin{array}{l}\text { Orientación al mercado proactiva (Fuente: Narver et al., 2004) }{ }^{\text {b }} \\
\text { Ayudamos a nuestros clientes a anticiparse a las tendencias en sus mercados } \\
\text { Continuamente intentamos descubrir necesidades adicionales de los clientes, aun de } \\
\text { las que ellos son inconscientes } \\
\text { En nuestros nuevos productos y servicios incorporamos soluciones a necesidades de } \\
\text { nuestros clientes con las que ellos no contaban } \\
\text { Buscamos nuevas formas como nuestros clientes utilizan nuestros productos y servicios } \\
\text { Buscamos oportunidades en áreas donde nuestros clientes encuentran difícil expresar } \\
\text { sus necesidades } \\
\text { Trabajamos de cerca con nuestros usuarios líderes, quienes intentan reconocer las ne- } \\
\text { cesidades de los compradores meses o años antes que la mayoría de los competidores } \\
\text { Extrapolamos las tendencias clave, para ganar comprensión acerca de lo que los mer- } \\
\text { cados actuales necesitarán en el futuro }\end{array}$ & $\begin{array}{l}0,66 \\
0,76 \\
0,72 \\
0,74 \\
0,60 \\
0,56 \\
0,58\end{array}$ & $\begin{array}{r}11,96 \\
14,71 \\
13,52 \\
14,12 \\
10,76 \\
9,84 \\
10,34\end{array}$ & $\begin{array}{l}C R=0,84 \\
\alpha=0,743\end{array}$ \\
\hline $\begin{array}{l}\text { Orientación al aprendizaje de mercado (Fuente: Weerawardena et al., 2006) } \\
\text { Su empresa recoge abundante información sobre sus mercados } \\
\text { Busca ideas innovadoras a través de la información del mercado } \\
\text { Obtiene abundante conocimiento sobre los segmentos de mercado } \\
\text { Obtiene conocimiento sobre los competidores } \\
\text { Revisa fracasos anteriores en los mercados } \\
\text { Analiza los efectos de los cambios en el mercado sobre su competitividad }\end{array}$ & $\begin{array}{l}0,72 \\
0,84 \\
0,88 \\
0,64 \\
0,57 \\
0,60\end{array}$ & $\begin{array}{l}13,70 \\
17,09 \\
18,63 \\
11,85 \\
10,33 \\
10,81\end{array}$ & $\begin{array}{l}C R=0,86 \\
\alpha=0,853\end{array}$ \\
\hline $\begin{array}{l}\text { Orientación al aprendizaje interno (Fuente: Weerawardena et al., 2006) }{ }^{\mathrm{b}} \\
\text { En su empresa se realizan importante actividades internas en I+D } \\
\text { El conocimiento generado en la I+D interna se comparte internamente } \\
\text { Se revisan los fracasos anteriores en I+D } \\
\text { El personal involucrado en I+D tiene gran nivel de habilidad } \\
\text { Se dedican importantes recursos económicos a I+D } \\
\text { El conocimiento obtenido en I+D se usa en las innovaciones } \\
\text { La propiedad intelectual de la empresa es importante } \\
\text { La capacidad para adquirir conocimiento en su empresa a través de I+D es alta }\end{array}$ & $\begin{array}{l}0,84 \\
0,83 \\
0,84 \\
0,83 \\
0,82 \\
0,85 \\
0,76 \\
0,84\end{array}$ & $\begin{array}{l}17,72 \\
17,26 \\
17,77 \\
17,31 \\
17,09 \\
17,99 \\
15,23 \\
17,52\end{array}$ & $\begin{array}{l}C R=0,94 \\
\alpha=0,945\end{array}$ \\
\hline
\end{tabular}

Estadísticos de ajuste para el modelo de medida de 35 indicadores para cinco constructos: $\chi_{(550)}^{2}=1374,15 ; \mathrm{GFI}=0,79$; RMSEA= 0,071; SRMR $=0,067 ; \mathrm{CFI}=0,95 ;$ TLI $(\mathrm{NNFI})=0,95 .{ }^{\mathrm{a}}$ composite reliability $\left(\rho_{\mathrm{c}}=\left(\sum \lambda_{\mathrm{i}}\right)^{2} \operatorname{var}(\xi) /\left[\left(\sum \lambda_{\mathrm{i}}\right)^{2} \operatorname{var}(\xi)+\sum \theta_{\mathrm{ii}}\right] ;(\mathrm{Bagozzi} \mathrm{y}\right.$ Yi, 1988). ${ }^{\mathrm{b}}$ extremos: $1=$ muy en desacuerdo; $7=$ muy de acuerdo. ${ }^{\mathrm{c}}$ extremos: $1=$ muy insatisfecho; $7=$ muy satisfecho. ${ }^{\mathrm{d}}$ Alpha de Cronbach.

Fuente: elaboración propia. 
Se utilizó un análisis factorial confirmatorio para evaluar la dimensionalidad, confiabilidad y validez de las escalas, conformado por cinco variables latentes. Los resultados también se muestran en el cuadro 1 . Dicho análisis mostró que cada uno de los 35 indicadores pesa significativamente en el factor destinado, lo cual indica validez convergente entre los ítemes de cada escala. Los indicadores de ajuste sugieren un ajuste razonable del modelo de los datos. También muestra que los constructos son confiables, la fiabilidad compuesta (CR) para los cinco constructos se encuentran entre 0,79 y 0,94 , todos excediendo el 0,7 , el cual es el nivel aceptable sugerido por Bagozzi y Yi (1988). El alfa de Cronbach también excede los mínimos recomendados de 0,7 .

El cuadro 2 presenta las correlaciones entre las variables latentes, sus valores medios y desviaciones típicas, así como, en la diagonal principal, la raíz cuadrada de la variancia media extraída (AVE). Los datos permiten comprobar que la raíz cuadrada del AVE para cada constructo es mayor que la correlación con cualquier otro constructo (Hulland,
1999). Esto satisface el criterio más exigente para poder aceptar la existencia de validez discriminante, de acuerdo con Fornell y Larcker (1981).

\subsection{Análisis y resultados}

Las hipótesis se contrastaron por medio de la estimación por mínimos cuadráticos ordinarios de la siguiente función de regresión lineal:

$$
\begin{gathered}
\mathrm{EXIN}=\beta \mathrm{O}+\beta_{1} \mathrm{OMR}+\beta_{2} \mathrm{OMP}+ \\
\beta_{3} \mathrm{OMR}^{2}+\beta_{4} \mathrm{OMP}^{2}+\beta_{5} \mathrm{OAM}+\beta_{6} \mathrm{OAI} \\
+\beta_{7} \mathrm{OMR} \times \mathrm{OAM}+\beta_{8} \mathrm{OMR} \times \mathrm{OAI}+ \\
\beta_{9} \mathrm{OMP} \times \mathrm{OAM}+\beta_{10} \mathrm{OMP} \times \mathrm{OAI}
\end{gathered}
$$

Donde, EXIN= Éxito de la innovación de la empresa; $O M R=$ Orientación al mercado reactiva; $O M P=$ Orientación al mercado proactiva; $O A M=$ Orientación al aprendizaje de mercado; OAI= Orientación al aprendizaje interno.

Dicho modelo es exhaustivo y se estima para minimizar los riesgos de no identificación en los conceptos relevantes. De este modo,

Cuadro 2. Matriz de correlaciones y estadísticos descriptivos

\begin{tabular}{|l|c|c|c|c|c|c|c|}
\hline \multicolumn{1}{|c|}{ Variable } & Media & $\begin{array}{c}\text { Desviación } \\
\text { estándar }\end{array}$ & 1 & 2 & 3 & 4 & 5 \\
\hline 1. Éxito de la innovación & 6,19 & 0,59 & 0,60 & & & & \\
\hline 2. Orientación al mercado reactiva & 5,78 & 0,74 & 0,33 & 0,62 & & & \\
\hline 3. Orientación al mercado proactiva & 5,61 & 0,86 & 0,26 & 0,55 & 0,66 & & \\
\hline 4. Orientación al aprendizaje de mercado & 5,56 & 0,97 & 0,33 & 0,49 & 0,48 & 0,71 & \\
\hline 5. Orientación al aprendizaje interno & 5,33 & 1,23 & 0,32 & 0,36 & 0,25 & 0,42 & 0,82 \\
\hline
\end{tabular}

Los valores de la diagonal corresponden a la raíz cuadrada de la varianza extraída para los constructos medidos con múltiples itemes. Fuente: elaboración propia. 
no sólo se introducen los términos que permiten contrastar las hipótesis planteadas en este trabajo sino otros términos que pudieran ejercer un efecto sobre la variable dependiente. Así, el modelo de regresión estimado es completo en cuanto a la descripción precisa de la forma en que la orientación al mercado y al aprendizaje influyen sobre el éxito de la innovación en la empresa. Para minimizar el riesgo de multicolinealidad que puede ser consecuencia de la presencia en la misma ecuación de efectos principales y efectos interacción utilizamos el método de centrado a la media recomendado por Jaccard et al. (1990) y Aiken y West (1991).

La contrastación de las hipótesis se lleva a cabo mediante análisis marginal propuesto por estos últimos autores. En concreto, la hipótesis 1 establece el sentido y forma del efecto de OMR sobre EXIN, proponiéndose un efecto positivo decreciente. Para contrastarlo, observamos que OMR influye EXIN de acuerdo con la siguiente función:

$$
\frac{\partial E X I N}{\partial O M R}=b_{1}+2 b_{3} O M R+b_{7} O A M+b_{8} O A I
$$

Como la hipótesis 1 no establece efecto moderador de las orientaciones al aprendizaje de la empresa, fijamos los valores de OAM y OAI en el valor medio obtenido. Como a su vez dichas variables están centradas a la media, dicho valor es nulo. Esto hace que el efecto de OMR sobre EXIN sea igual a:

$$
\frac{\partial E X I N}{\partial O M R}=b_{1}+2 b_{3} O M R
$$

Sustituyendo los valores de los coeficientes del cuadro 3 observamos que para valores bajos de OMR (-1 desviación típica de OMR= $-0,741$, ver cuadro 2), el efecto de OMR sobre EXIN es de $0,154+2 \times(-0,037) \times-0,741$. Esto da un valor final del efecto igual a 0,208 , que siguiendo los cálculos sugeridos por Aiken y West (1991) ofrece un valor $\mathrm{t}=2,223$ $(\mathrm{p}<0,05)$. Para valores altos de $\operatorname{OMR}(0,741$, ver cuadro 2) el efecto de OMR sobre EXIN es de $0,099(t=0,816 ; p>0,1)$. Los resultados obtenidos muestran un efecto positivo y significativo de la orientación al mercado reactiva en valores bajos. El cambio de efecto $(-0,109)$ muestra una $\mathrm{t}=-0,447(\mathrm{p}>0,1)$. Por tanto, podemos concluir que si bien el efecto es positivo, no se observa saturación, por lo que aceptamos H1 sólo de modo parcial.

Para contrastar la hipótesis 2 que establece el sentido y forma del efecto de OMP sobre EXIN y con la misma orientación del análisis de la hipótesis 1 se establece que dicho efecto sea igual a:

$\frac{\partial E X I N}{\partial O M P}=b_{2}+2 b_{4} O M P$

Sustituyendo los valores de los coeficientes del cuadro 3 observamos que para valores bajos de OMP (-1 desviación típica de OMP= $-0,859$, ver cuadro 2), el efecto de OMP sobre EXIN es de $-0,029+2 \times(-0,106) \times-0,859$. Esto da un valor final del efecto igual a 0,153 , que siguiendo los cálculos sugeridos por Aiken y West (1991) ofrece un valor $\mathrm{t}=3,052$ $(\mathrm{p}<0,05)$. Para valores altos de $\operatorname{OMP}(0,859$, ver cuadro 2) el efecto de OMP sobre EXIN 
es de $-0,211(t=-2,178 ; p<0,05)$. Los resultados obtenidos muestran un efecto positivo y significativo de la orientación al mercado proactiva en valores bajos y un efecto negativo significativo en valores altos. El cambio de efecto $(-0,36$, ver gráfica 1$)$ muestra una $\mathrm{t}=-3,35(\mathrm{p}<0,01)$. Por tanto, podemos concluir que existe un efecto de saturación, por lo que aceptamos $\mathrm{H} 2$.

En cuanto al efecto directo de las orientaciones al aprendizaje sobre los resultados de la innovación se puede observar que ambos tipos de orientación ejercen un efecto positivo y altamente significativo. Aunque esto no forma parte de las hipótesis, centradas en la orientación al mercado y sus interacciones con las orientaciones al aprendizaje, el resultado está en línea con la literatura que muestra la importancia del aprendizaje en la mejora de los procesos de innovación (Sinkula et al., 1997; Weerawardena et al., 2006).

Respecto a la moderación de la orientación al aprendizaje de mercado (OAM) (H3) y de la orientación al aprendizaje interno $(\mathrm{H} 4)$ en el efecto de OMP sobre EXIN, los resultados obtenidos y presentados en el cuadro 3 sugieren que sólo la orientación al aprendizaje de mercado tiene potencial para presentar un efecto significativo. El coeficiente obtenido para la interacción de OMP con OAM es positivo y significativo, lo que está en línea con la hipótesis propuesta. No obstante, para asegurar la significatividad total del efecto moderación es necesario comprobarlo mediante análisis marginal (Aiken y West, 1991). En la gráfica 1 se aprecia el efecto de OMP sobre EXIN. Asimismo se indica cuál es el efecto en cada nivel de OMP y OAM.

Los resultados ahí presentados permiten comprobar que tanto en niveles bajos como altos de OAM el efecto de OMP sobre EXIN siempre es menor cuando OMP es alto que cuando es bajo. Esto es prueba del cumplimiento de la Hipótesis 2 en cualquiera de los niveles de OAM. Por otra parte, para contrastar la Hipótesis 3, se observa que el efecto de OMP sobre EXIN siempre es ma-

\section{Cuadro 3. Resultados del análisis de regresión}

\begin{tabular}{|c|c|}
\hline $\begin{array}{l}\text { Variable dependiente: } \\
\text { Éxito de la Innovación }\end{array}$ & $\begin{array}{l}\text { Coeficiente no } \\
\text { estandarizado }\end{array}$ \\
\hline Constante & $6,194(145,690)$ \\
\hline \multicolumn{2}{|l|}{ Variables independientes } \\
\hline Orientación al mercado reactiva & $0,154(2,71)^{\star * *}$ \\
\hline Orientación al mercado proactiva & $-0,029(-0,578)$ \\
\hline Orientación al mercado reactiva ${ }^{2}$ & $-0,037(-0,597)$ \\
\hline Orientación al mercado proactiva ${ }^{2}$ & $-0,106(-3,595)^{\star * *}$ \\
\hline $\begin{array}{l}\text { Orientación al aprendizaje de mer- } \\
\text { cado }\end{array}$ & $0,150(3,532)^{\star * *}$ \\
\hline Orientación al aprendizaje interno & $0,082(2,781)^{\star * *}$ \\
\hline \multicolumn{2}{|l|}{ Términos de interacción } \\
\hline $\begin{array}{l}\text { Orientación al mercado reactiva } \\
\text { x Orientación al aprendizaje de } \\
\text { mercado }\end{array}$ & $0,038(0,610)$ \\
\hline $\begin{array}{l}\text { Orientación al mercado reactiva x } \\
\text { Orientación al aprendizaje interno }\end{array}$ & $0,046(0,867)$ \\
\hline $\begin{array}{l}\text { Orientación al mercado proactiva } \\
\text { x Orientación al aprendizaje de } \\
\text { mercado }\end{array}$ & $0,161(3,490)^{\star * *}$ \\
\hline $\begin{array}{l}\text { Orientación al mercado proactiva x } \\
\text { Orientación al aprendizaje interno }\end{array}$ & $0,002(0,044)$ \\
\hline$R^{2}$ & 0,254 \\
\hline$R^{2}$ Ajustado & 0,227 \\
\hline
\end{tabular}




\section{Gráfica 1}

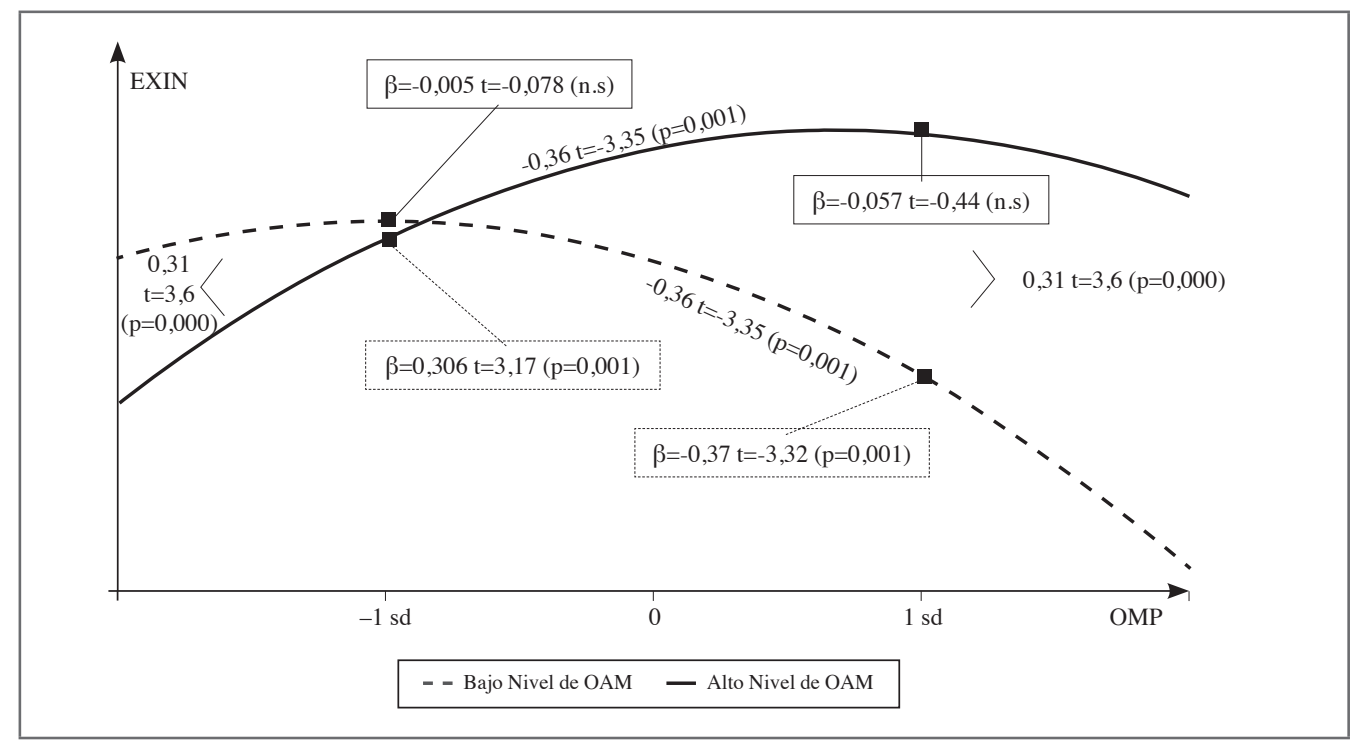

Fuente: elaboración propia.

yor en presencia de altos niveles de OAM. Además, la diferencia de efecto entre valores altos y bajos de OAM es de 0,31, que obtiene un valor $\mathrm{t}=3,6(\mathrm{p}<0,01)$. Por lo tanto, efectivamente se observa que OAM modera positivamente el efecto de OMP sobre EXIN. Esto nos permite aceptar H3.

\section{Discusión}

Los resultados obtenidos del modelo teórico probado en este estudio son consistentes con otros estudios que incorporan el efecto sinérgico de la orientación al aprendizaje y la orientación al mercado sobre el éxito de la innovación (Atuahene-Gima et al., 2005; Weerawardena et al., 2006). Aporta nuevas señales en el conocimiento de cómo una empresa orientada al aprendizaje puede mejorar los comportamientos orientados al mercado que procuren una ventaja competitiva desde la mirada del éxito de los procesos de innovación. Este artículo examina los efectos lineales y no lineales de la orientación al mercado reactiva y proactiva sobre el éxito de la innovación. Los pocos trabajos empíricos sobre estas dos dimensiones de la orientación al mercado no han explorado la relación compleja y sinérgica con la orientación al aprendizaje; esto le da relevancia al presente estudio. Soporta el argumento de que la orientación al mercado está compuesta por una dimensión reactiva, en la cual se ha centrado la mayoría de los estudios de orientación al mercado (Narver et al., 2004) y una orientación proactiva. Esto queda evidenciado en la medida en que ambas dimensiones pueden tener efectos diferentes sobre el éxito de la innovación y que dicho efecto está condicionado por una orientación al aprendizaje, ayudando en la comprensión del debate del detrimento o 
impulso de la innovación cuando se implementa una orientación al mercado.

En particular, la relación entre la orientación al mercado reactiva y el éxito de la innovación es lineal y positiva, sugiriendo que las empresas preocupadas por conocer y entender las necesidades actuales de los clientes y que innovan de acuerdo con ellas siempre obtienen resultados positivos. Respecto a la no observación de un efecto saturación en el efecto de la orientación al mercado reactiva, a pesar de que el efecto cuadrático fue negativo, no fue significativo. Esto puede explicarse por el contexto tecnológico presente en las economías latinoamericanas y en particular en la colombiana. Tsai et al. (2008) demostraron que un efecto lineal y no cuadrático de la relación entre la orientación al mercado reactiva y el éxito de la innovación es observable en entornos tecnológicamente estables. En otras palabras, en condiciones de bajas tasas de cambio tecnológico no se dan los efectos potenciales negativos asociados con niveles altos de orientación al mercado reactiva. En Colombia el entorno tecnológico no es turbulento, sino más bien estable. Malaver y Vargas (2004, p. 18) señalan para el caso colombiano que: "Las empresas no se han organizado para manejar la tecnología, y los avances en materia de uso, explotación y desarrollo de las tecnologías incorporadas por las empresas... explican el puntaje medio bajo que reciben las capacidades tecnológicas... no obstante de ser innovadoras".

La relación entre la orientación al mercado proactiva y el éxito de la innovación presenta un efecto saturación. Aunque las empresas que son capaces de aprovechar y utilizar la información y el conocimiento obtenido de indagar las necesidades latentes de los clientes tienen hasta cierto punto mejores capacidades y rendimiento en los resultados de la innovación, los teóricos señalan que un comportamiento proactivo excesivo puede ser dañino (Levinthal y March, 1993), resultado contrastado empíricamente en el presente estudio. Esto es así dado que un alto nivel de orientación proactiva al mercado exige a las empresas muchos proyectos de tipo exploratorio que generalmente requiere de nuevos y específicos conocimientos no basados en la experiencia siendo necesario invertir en estructuras organizacionales diferentes a las existentes implicando altos riesgos y costos. $\mathrm{Al}$ asumir estas nuevas formas organizativas las empresas aumentan las posibilidades de asumir errores e ineficiencias operativas que pueden repercutir de manera negativa en los resultados de la innovación. Además, pueden existir barreras cognitivas para el procesamiento de nueva información de mercado tales como la frecuencia y cantidad de tiempo que se requeriría en su difusión generando costos más altos que los beneficios esperados (Maltz y Kohli, 1996).

Por otro lado, se probó que las capacidades de aprendizaje de la firma desempeñan un papel importante en la generación de la innovación (Sinkula et al., 1997). Así, un set de orientaciones al aprendizaje debe ser prerrequisito del éxito de la innovación (Weerawardena et al., 2006). En este estudio se observa cómo uno de los conductores de los procesos de innovación en una empresa es su aprendizaje. Aquí se enfatiza que dicho aprendizaje proviene de fuentes internas y externas. El efecto directo del aprendizaje 
tanto de fuentes de mercado como de fuentes internas es significativo y positivo. Este hallazgo es una importante extensión del punto de vista del rol del aprendizaje como un recurso intangible de la empresa en la construcción de su ventaja competitiva (Baker y Sinkula, 1999b; Day, 1994a; Dickson, 1996; Hunt y Morgan, 1996).

Este estudio propone que el efecto sinérgico entre el aprendizaje y la orientación al mercado es complejo. Las empresas que toman la decisión de comportarse orientadas al mercado de una manera proactiva deben tener en consideración que hay bastantes posibilidades de fracaso, es decir, que demasiadas actividades de exploración pueden hacer que se pierda el foco de las capacidades centrales desarrolladas por la empresa. Este estudio, a diferencia de Baker y Sinkula (1999b), enfatiza que la orientación al aprendizaje del mercado, promueve de manera positiva el efecto saturado de la orientación al mercado proactiva. Las empresas que adquieran niveles altos de aprendizaje con fuentes del mercado buscarán información más allá de sus sistemas formales de información establecidos y sus directivos asegurarán que sus redes incluyan personas con diferentes perspectivas en comparación con aquellas que prevalecen en la organización. Con una fuerte orientación al aprendizaje de mercado las empresas pueden incorporar comportamientos orientados al mercado de manera proactiva permitiéndoles un mayor éxito en los procesos de innovación sin olvidar su correspondiente efecto de saturación.

En cuanto a la orientación al aprendizaje interno, los directivos no deben olvidar que la memoria organizacional (conocimiento de fuentes internas) es particularmente importante. El grado con el cual ese conocimiento interno sea usado determinará también el éxito de los procesos de innovación. En otras palabras, nuevos procedimientos o capacidades adquiridas por una orientación al aprendizaje pueden ser más efectivos pero la empresa no debe rechazar las capacidades en las cuales ha invertido fuertemente. Sin embargo, para el caso de nuestro estudio el efecto moderador del aprendizaje basado en fuentes internas sobre la relación de la orientación al mercado (proactiva y reactiva) y el éxito de la innovación no ha sido significativo. Este hecho se puede explicar porque el aprendizaje de fuentes internas es un tipo de aprendizaje adaptativo (Dess et al., 2003), es decir, que las empresas utilizan el conocimiento interno y privado que poseen. Una falla potencial del aprendizaje adaptativo es que éste ocurre dentro del contexto de los modelos mentales existentes en la empresa (Akgun et al., 2006; Slater y Narver, 1995) y la eficacia del procesamiento de la información de mercado depende del grado en que dichos modelos mentales utilizados para interpretar dicha inteligencia de mercado se ajusten a la realidad, sean exactos y sean compartidos por toda la organización (Day, 1991; Senge, 1990). Si los modelos mentales son imperfectos, el aprendizaje de fuentes internas puede no producir un efecto óptimo sobre el cambio en las maneras como la organización comprende el qué hacer, inhibiendo la creación de valor a la innovación. En el caso colombiano las decisiones relacionadas por ejemplo con el cambio tecnológico son el resultado de procesos informales y no producto de la I+D y 
su capacidad de absorción, sino más bien, resultados de decisiones de inversión que toman los directivos y responden a necesidades puntuales y de carácter funcional. El manejo informal de la tecnología es un factor que limita el desarrollo de la innovación (Malaver y Vargas, 2004).

\section{Conclusiones}

La primera contribución de este artículo es la conceptualización y examen de un modelo integrado que especifica las relaciones entre las dimensiones proactiva y reactiva de la orientación al mercado con el éxito de la innovación y la orientación al aprendizaje de la empresa desde la perspectiva de sus fuentes de información. El éxito de la innovación en este estudio no se reduce a una medida de desempeño de la innovación o a una salida relacionada con la cantidad de innovación de producto, sino que se contempla el éxito de una cultura innovadora general de la firma.

Los resultados sugieren que los beneficios de las dimensiones proactiva y reactiva de la orientación al mercado se despliegan de manera independiente más que integradora. Este resultado obtenido en una muestra de empresas operando en una economía en desarrollo sugiere con respecto al éxito de la innovación que las dos vertientes reactiva y proactiva incrementan el poder explicativo de la tradicional orientación al mercado. En este contexto, se privilegia un éxito de la innovación más satisfactorio para una orientación reactiva del mercado que para una orientación proactiva; resultado opuesto al de Narver et al. (2004). A diferencia de otros estudios relacionados con las dos di- mensiones del mercado, en este trabajo los efectos del aprendizaje se tienen en cuenta para observar el éxito de la innovación. La orientación al aprendizaje de mercado y al aprendizaje interno, además de tener un efecto directo sobre el éxito de la innovación, en el primer caso favorece el éxito de la orientación al mercado proactiva.

\subsection{Limitaciones y dirección de futura investigación}

Este artículo está sujeto a las limitaciones usuales y propias de estudios transversales, en particular al uso de informantes individuales y medidas subjetivas. Sin embargo, como este estudio lo mostró los informantes estaban bien calificados para reportar la información de las variables estudiadas. Un estudio longitudinal arrojaría óptimos comportamientos de las empresas con relación al desempeño y el énfasis de los diferentes tipos de orientación al mercado y el aprendizaje. Adicionalmente se sugiere para futuros estudios la inclusión de variables de control reconocidas en la literatura de innovación a fin de reducir la posibilidad de explicaciones alternativas.

A pesar de dicha limitación este estudio hace importantes contribuciones para futuras investigaciones. Previos estudios han identificado un efecto de interacción entre la orientación al mercado reactiva y proactiva. Sin embargo empíricamente dicha hipótesis sigue sin demostrarse. Futuros desarrollos podrían abordar este tópico para ver si la orientación al mercado reactiva y proactiva se complementan. Existe aún poca evidencia de los antecedentes de estas dos dimensio- 
nes de la orientación al mercado, así como estudios que analicen de manera simultánea efectos moderadores internos y externos a la organización. En conclusión, siguen siendo escasos los estudios empíricos que analizan las relaciones de las dos dimensiones de la orientación al mercado sobre la innovación.

Los resultados de este estudio son propios de una economía en desarrollo, con lo cual, más allá de ser una limitación brinda una oportunidad de conocer los fenómenos en un contexto habitualmente resuelto en economías desarrolladas.

\section{Referencias}

Aiken, L. S., and West, S. G. (1991). Multiple Regression: Testing and interpreting interactions. Thousand Oaks, California: Sage Publications, Inc.

Akgun, A. E., Lynn, G. S., and Byrne, J. C. (2006). Antecedents and consequences of unlearning in new product development teams. Journal of Product Innovation Management, 23 (1), 73-88.

Anderson, J. C., and Gerbing, D. W. (1988). Structural equation modeling in practice: A review and recommended two-step approach. Psychological bulletin, 103 (3), 411-423.

Atuahene-Gima, K. (1995). An exploratory analysis of the impact of market orientation on new product performance: A contingency approach. Journal of Product Innovation Management, 12 (4), 275-293.

Atuahene-Gima, K., Slater, S. F., and Olson, E. M. (2005). The contingent value of responsive and proactive market orientations for new product program performance. Journal of Product Innovation Management, 22 (6), 464-482.
Bagozzi, R. P., and Yi, Y. (1988). On the evaluation of structural equation models. Journal of the Academy of Marketing Science, 16 (1), 74-94.

Baker, W. E., and Sinkula J. M. (1999a). Learning orientation, market orientation, and innovation: Integrating and extending models of organizational performance. Journal of Market Focused Management, 4 (4), 295-308.

Baker, W. E., and Sinkula J. M. (1999b). The synergistic effect of market orientation and learning orientation on organizational performance. Journal of the Academy of Marketing Science, 27 (4), 411-427.

Baker, W. E., and Sinkula, J. M. (2002). Market orientation, learning orientation and product innovation: Delving into the organization's black box. Journal of Market Focused Management, 5 (1), 5-23.

Baker, W. E., and Sinkula, J. M. (2007). Does market orientation facilitate balanced innovation programs? An organizational learning perspective. Journal of Product Innovation Management, 24 (4), 316-334.

Baker, W. E., and Sinkula, J. M. (2009). The complementary effects of market orientation and entrepreneurial orientation on profitability in small businesses. Journal of Small Business Management, 47 (4), 443-464.

Boumgarden, P., Nickerson, J., and Zenger, T. R. (2012). Sailing into the wind: Exploring the relationships among ambidexterity, vacillation, and organizational performance. Strategic Management Journal, 33 (6), 587-610.

Cavusgil, S. T., and Elvey-Kirk, L. A. (1998). Mail survey response behavior: a conceptualization of motivating factors and an empirical study. European Journal of Marketing, 32 (11/12), 1165-1192.

Christensen, C. M., and Bower, J. L. (1996). Customer power, strategic investment, and the failure 
of leading firms. Strategic Management Journal, 17, 197-219.

Cohen, W. M., and Levinthal, D. M. (1989). Innovation and learning: The two faces of R\&D. The Economic Journal, 99 (397), 569-596.

Cohen, W. M., and Levinthal, D. M. (1990). Absorptive capacity: A new perspective on learning and innovation. Administrative Science Quarterly, 35 (1), 128-152.

Cyert, R. M., and March, J. G. (1963). Behavioral theory of the firm. Cambridge, MA: Blackwell Business.

Danneels, E., and Kleinschmidt, E. J. (2001). Product innovativeness from the firm's perspective: Its dimensions and their relation with project selection and performance. Journal of Product Innovation Management, 18 (6), 357-373.

Day, G. S. (1991). Learning about markets. Marketing Science Institute, Report Number 91-117, Cambridge, MA: Marketing Science Institute.

Day, G. S. (1994a). Continuous learning about markets. California Management Review, 36 (4), 9-31.

Day, G. S. (1994b). The capabilities of marketdriven organizations. The Journal of Marketing, 58 (4), 37-52.

Deshpande, R., Farley, J., and Webster, F. (1993). Corporate culture, customer orientation, and innovativeness in Japanese firms: A Quadrat Analysis. Journal of Marketing, 57 (1), 23-37.

Dess, G. G., Ireland, R. D., Zahra, S. A., Floyd, S. W., Janney, J. J., and Lane, P. J. (2003). Emerging issues in corporate entrepreneurship. Journal of Management, 29 (3), 351-378.

Dickson, P. R. (1996). The static and dynamics mechanics of competition: A comment on Hunt and Morgan's comparative advantage theory. Journal of Marketing, 1, 93-122.

Dixon, N. M. (1992) Organizational learning: A review of literature with implications for HRD professionals. Human Resource Development Quarterly, 3 (1), 29-49.

Fornell, C., and Larcker, D. F. (1981). Evaluating structural equation models with unobservable variables and measurement errors. Journal of Marketing Research, 18 (1), 39-50.

Frosch, R. (1996). The customer for R\&D is always wrong! Research-Technology Management, 39 (6), 22-27.

Garcia, R., and Calantone, R. (2002). A critical look at technological innovation typology and innovativeness terminology: A literature review. Journal of Product Innovation Management, 19 (2), 110-132.

Grinstein, A. (2006). The relationship between market orientation and its components on innovation consequences: A meta-analysis. Journal of The Academic Marketing Science, 36, 166-173.

Grinstein, A. (2008). The effect of market orientation and alternative strategic orientations: A meta-analysis. European Journal of Marketing, 42 (1/2), 115-134.

Hamel, G., and Prahalad, C. K. (1994). Competing for the future. Boston: HBS Press.

Han, J. K., Namwoon, K., and Srivastava R. K. (1998). Market orientation and organizational performance: Is innovation a missing link? Journal of Marketing, 62 (4), 30-45.

Huber G. (1991). Organizational learning: The contributing processes and literature. Organization Science, 2, 88-115.

Hulland, J. (1999). Use of partial least squares (PLS) in strategic management research: A review of four recent studies. Strategic Management Journal, 20 (2), 195-202.

Hult, G., Thomas, M., Hurley, R. F., and Knight, G. A. (2004). Innovativeness: Its antecedents and impact on business performance. Industrial Marketing Management, 33 (5), 429-438. 
Hunt, S. D., and Morgan, R. M. (1995). The comparative advantage theory of competition. Journal of Marketing, 59 (April), 1-15.

Hunt, S. D., and Morgan, R. M. (1996). The resource - advantage theory of competition: Dynamics, path dependencies and evolutionary dimensions. Journal of Marketing, 60 (3), 107-114.

Hurley, R. F., Hult, G. T., and Knight, G. A. (2005). Innovativeness and capacity to innovate in a complexity of firm-level relationships: A response to Woodside 2004. Industrial Marketing Management, 34 (3), 281-283.

Hyvarinen L. (1990). Innovativeness and its indicators in small and medium-sized industrial enterprises. International Small Business Journal, 9 (1), 64-74.

Jaccard, J., Turrisi, R., and Wan, C. K. (1990). Interaction effects in multiple regression. Newbury Park, CA: Sage Publications.

Jaworski, B., and Kohli, A. (1993). Market orientation: Antecedents and consequences. Journal of Marketing, 57 (3), 53-70.

Kim, Y., Song, K., and Lee J. (1993). Determinants of technological innovation in the small firms in Korea. $R \&$ D Management, 23 (3), 215-226.

Kohli, A. K., and Jaworski, B. J. (1990). Market orientation: The construct, research propositions, and managerial implications. Journal of Marketing, 54 (2), 1-18.

Lamore, P. R., Berkowitz, D., and Farrington, P. A. (2013). Proactive/responsive market orientation and marketing - research and development integration. Journal of Product Innovation Management, 30 (4), 695-711.

Lee, Y., and O'Connor, G. C. (2003). The impact of communication strategy on launching new products: The moderating role of product innovativeness. Journal of Product Innovation Management, 20 (1), 4-21.
Levinthal, D. A., and March, J. G. (1993). The myopia of learning. Strategic Management Journal, 14, 95-112.

Li, C. R., Lin, C. J., and Chu, C. P. (2008). The nature of market orientation and the ambidexterity of innovations. Management Decision, 46, 1002-1026.

Li, T., and Calantone, R. (1998). The impact of market knowledge competence on new product advantage: Conceptualization and empirical examination. Journal of Marketing, 62 (4), 13-29.

Lukas, B. A., and Ferrell, O. C. (2000). The effect of market orientation on product innovation. Journal of the Academy of Marketing Science, 28 (2), 239-248.

Malaver, R. F., y Vargas, M. (2004). Los procesos de innovación en América Latina: aportes para su caracterización. Academia. Revista Latinoamericana de Administración, 33, 5-33.

Maltz, E., and Kohli, A. K. (1996). Market intelligence dissemination across functional boundaries. Journal of Marketing Research, 33, 47-61.

March, J. G. (1991). Exploration and exploitation in organizational learning. Organization Science, 2 (1), 71-87.

Moorman, C., and Rust, R. (1999). The role of marketing. Journal of Marketing, 63, 180-197.

Narver, J. C., and Slater, S. F. (1991). Becoming more market oriented: An exploratory study of the programmatic and market-back approaches. Working paper, Marketing Science Institute.

Narver, J. C., Slater, S. F., and Maclachlan, D. L. (2004). Responsive and proactive market orientation and new product success. Journal of Product Innovation Management, 21, 334-347.

Nickerson, J. A., and Zenger, T. R. (2002). Being efficiently fickle: A dynamic theory of organizational choice. Organization Science, 13 (5), 547-566. 
Olsen, S. B. (2009). Choosing between internet and mail survey modes for choice experiment surveys considering non-market goods. Environmental and Resource Economics, 44 (4), 591-610.

Pelham, A., and Wilson, D. (1996). A longitudinal study of the impact of market structure, firm structure, strategy, and market orientation culture on dimensions of business performance. Journal of the Academy of Marketing Science, 24 (1), 27-43.

Ruvio, A.A., Shoham, A., Vigoda-Gadot, E., and Schwabsky, N. (2014). Organizational Innovativeness: Construct Development and Cross-Cultural Validation. Journal of Product Innovation Management, 31 (5), 1004-1022.

Senge, P. M. (1990). The fifth discipline: The art and practice of the learning organization. New York: Doubleday.

Simpson, P. M., Siguaw, J. A., and Enz, C. A. (2006). Innovation orientations outcomes: The good and the bad. Journal of Business Research, 59 (10), 1133-1141.

Sinkula, J. M., Baker, W., and Noordewier, T. G. (1997). A framework for market-based organizational learning: Linking values, knowledge and behavior. Journal of the Academy of Marketing Science, 25, 305-318.

Slater, S. F., and Narver, J. C. (1994). Does competitive environment moderate the market orientation-performance relationship? Journal of Marketing, 58 (1), 46-55.

Slater, S. F., and Narver, J. C. (1995). Market orientation and the learning organization, The Journal of Marketing, 59 (3), 1652-1669.

Slater, S. F., and Narver, J. C. (1998). Customerled and market-oriented: Let's not confuse the two. Strategic Management Journal, 19 (10), 1001-1006.
Slater, S. F., and Narver, J. C. (1999). Market-oriented is more than being customer-led. Strategic Management Journal, 20 (12), 1165-1170.

Song, M., and Thieme, R. J. (2006). A cross-national investigation of the R\&D-marketing interface in the product innovation process. Industrial Marketing Management, 35, 308-322.

Tan, M., and Liu, Z. (2014). Paths to success: An ambidexterity perspective on how responsive and proactive market orientations affect SME's business performance. Journal of Strategic Marketing, 22 (5), 420-441.

Totterdell, P., Desmond, L., Kamal, B., Chris, C., and Toby, W. (2002). An investigation of the contents and consequences of major organizational innovations. International Journal of Innovation Management, 6, 343-368.

Tsai, K. H., Chou, C., and Kuo, J. H. (2008). The curvilinear relationship between responsive and proactive market orientation and new product performance: A contingent link. Industrial Marketing Management, 37, 884-894.

Voola, R., and O'Cass, A. (2010). Implementing competitive strategies: The role of responsive and proactive market orientations. European Journal of Marketing, 44 (1/2), 245-266.

Webster, F. E. Jr. (1992). The changing role of marketing in the corporation. Journal of Marketing, 56 (October), 1-17.

Weerawardena, J., O'Cass, A., and Julian, C. (2006). Does industry matter? Examining the role of industry structure and organizational learning in innovation and brand performance. Journal of Business Research, 59, 37-45.

Yannopoulos, P., Auh, S., and Menguc, B. (2012). Achieving fit between learning and market orientation: Implications for new product performance. Journal of Product Innovation Management, 29 (4), 531-545. 
Zhang, J., and Duan, Y. (2010). The impact of dif ferent types of market orientation on product innovation performance: Evidence from Chinese manufacturers. Management Decision, 48 (6), 849-867.

Zhao, Y., Li, Y., Lee, S. H., and Chen, L. B. (2011) Entrepreneurial orientation, organizational learning, and performance: Evidence from China. Entrepreneurship Theory and Practice, 35 (2), 293-317. 
